\title{
A Go-NoGo Gauge for USP Apparatus 1 Baskets
}

\author{
Kevin Coleman Bynum ${ }^{1}$, Abe S. Kassis ${ }^{2,3}$, and Tarun Patel ${ }^{2}$ \\ 'Boehringer Ingelheim Pharmaceuticals, Inc., 900 Ridgebury Rd., Ridgefield, CT 06877 \\ ${ }^{2}$ Novartis Pharmaceuticals, One Health Plaza, East Hanover, NJ 07936
}

e-mail:abe.kassis@novartis.com

\begin{abstract}
A simple, cost-effective, time-saving device has been invented to control the variability of a basket. This device not only controls the parameters set in USP $<711>$ (height, cylinder ID, and OD) but also controls the cylinder symmetry, which is not defined in USP $<711>$.
\end{abstract}

\section{INTRODUCTION}

D issolution testing is a requirement for all solid oral dosage forms and is used for drug product release and stability testing. The dissolution test is the most important analytical test for detecting physical changes in an API and in the formulation. The two commonly used dissolution apparatus are the basket (USP Apparatus 1) and the paddle (USP Apparatus 2). Both apparatus have been widely accepted by the pharmaceutical community for measuring the rate of dissolution of an API from a given pharmaceutical solid dosage form.

The setup of dissolution Apparatus 1 and 2 requires control over many variables as defined in USP <711> (1), European Pharmacopoeia 2.9.3 (2), and Japanese Pharmacopoeia 15 (3). The variables in both of these apparatus are vessels, shaft dimensions, shaft wobble, rotation speed, shaft height from the bottom of the vessel, vessel centering and tilt, temperature, leveling of the dissolution apparatus at its base, vibration, and so forth. Many literature articles describe methods to control these variables (4-23). However, none of these address control of basket variability (refer to USP $<711>$ ).

\section{USP and FDA Requirements for Basket Apparatus}

The basket apparatus consists of a wire-mesh basket that is attached to a rotation shaft, which is then immersed into a dissolution vessel for the duration of the dissolution test. Since the dosage unit is in direct contact with the basket, the physical dimensions and motion of the basket can have a dramatic effect on the dissolution rate of the solid dosage unit. Because of the critical nature of the basket, it is tightly controlled by several mechanisms. First, the dimensions of basket height, i.d. and o.d. of the basket opening, height of the open screen, and size of the mesh are specified in USP $<711>$. Next, the amount of wobble at the bottom of the basket while rotating is checked with a wobble meter at periodic intervals, anywhere from time of

${ }^{3}$ Corresponding author. use to once a year, to ensure that it is within the 1-mm specification indicated in USP $<711>$. Finally, a functional test using standardized performance-verification tablets is executed. The rate of release of the standardized calibrator tablet is measured and compared to the acceptance criteria. The performance verification tablets and acceptance criteria are designed so that if the apparatus is not set up in accordance with the tight USP specifications, it will not pass this final test.

\section{The Device}

To control variability from the basket itself, a device was invented to evaluate the critical dimensions of each basket. The device is shown in Figure 1 and consists of a center post with a bottom lip, which is attached to a square base. There is a back shield that is precisely spaced from the center post to exactly allow a USP-compliant basket to fit into the gauge. The basket is inverted and placed over the center post. The back shield of the gauge is designed in such a way that the basket will not fit down the center post if it is not perfectly cylindrical. Additionally, any slight imperfection in basket symmetry will be evident when the basket is spun on the post. A passing basket will spin freely with no resistance, while a failing basket will jam and will not spin freely. Furthermore, the height of the basket is checked by the shield. A basket with a proper height will fit flush against the bottom lip of the gauge and will not be taller than the shield. A USP-compliant basket is shown on the gauge in Figure 2. For a USP-compliant basket, the center pin will be flush against the bottom mesh of the basket, as shown in Figure 3.

A non-USP-compliant basket that is too short will not seat onto the lip, and a visible gap will be present. Likewise, a basket that is bent or is not a true cylinder will not fit down the post, as shown in Figure 4. Even a slight dent in the basket will cause it to bind between the precisely positioned shield and the center post when rotated. A basket that is too tall will not be flush with the top of the back shield, and when the bottom of the basket is compressed, it will not be flush against the top of the post, as shown in Figure 5. 


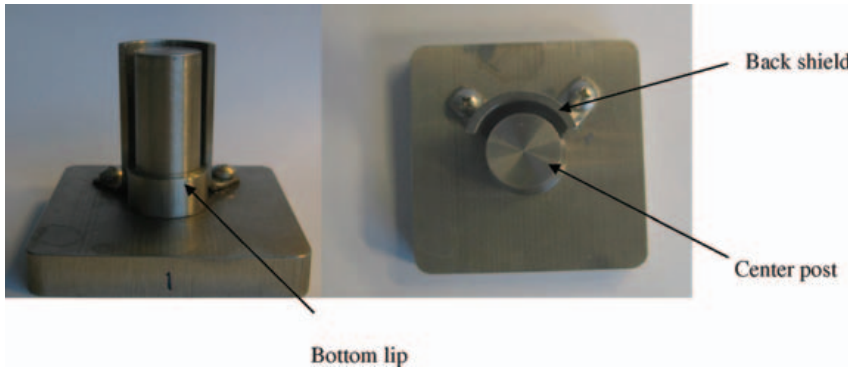

Figure 1. Front and top view of the Go-NoGo basket gauge.

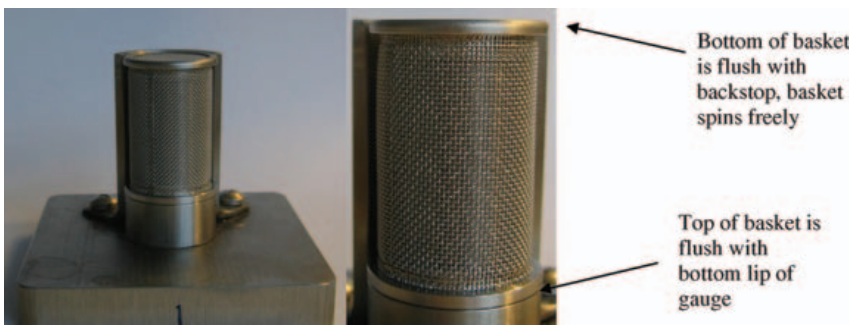

Figure 2. USP-compliant basket in the Go-NoGo gauge.

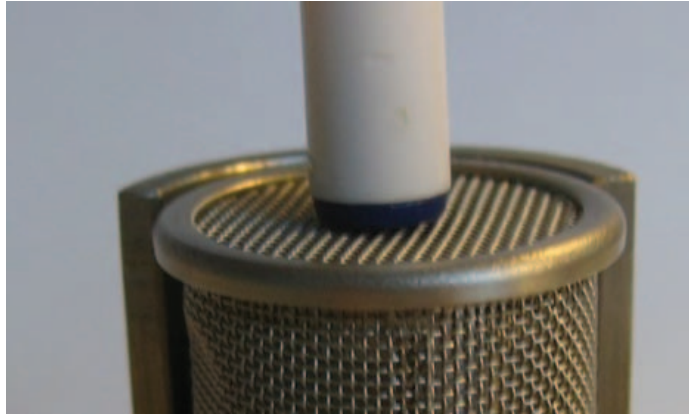

Figure 3. Basket bottom is flush against center post.

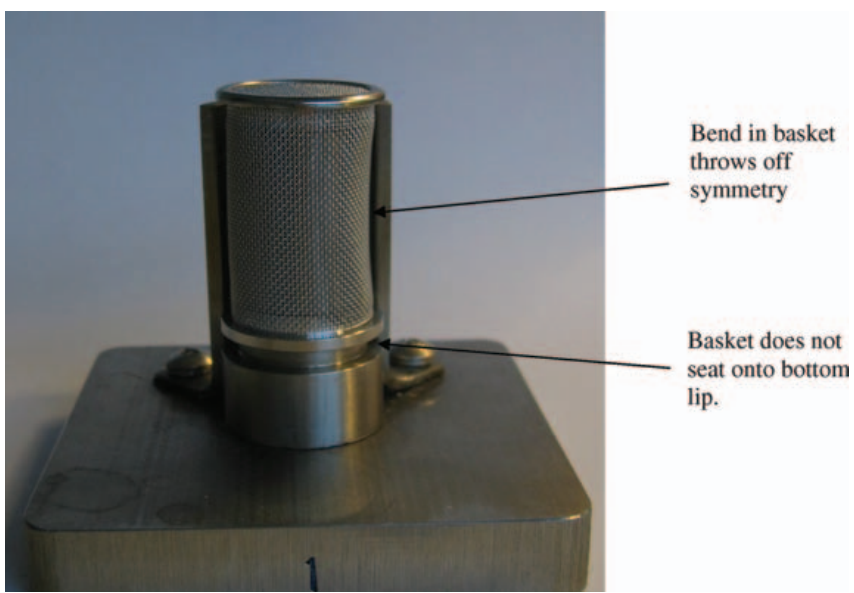

Figure 4. Non-USP-compliant basket.

Dissolution Technologies | NOVEMBER 2008

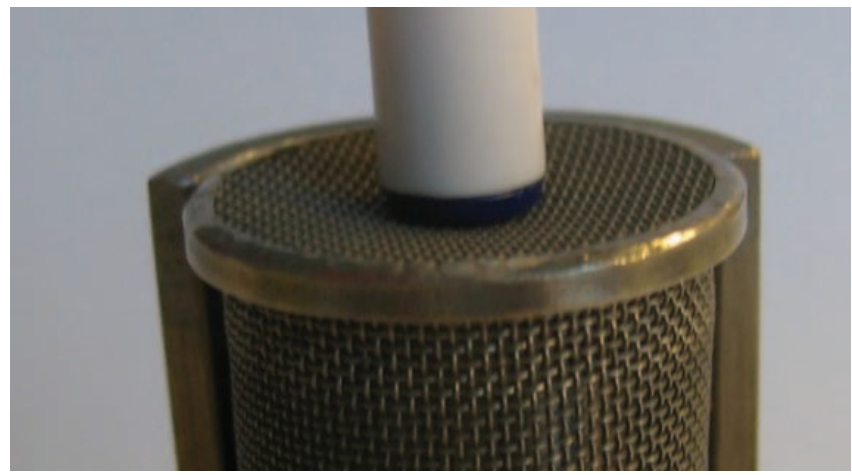

Figure 5. Non-USP-compliant basket (1-mm too tall); bottom of basket can be depressed.

\section{DISCUSSION}

The USP defines the precise measurements of the Apparatus 1 basket to ensure consistency in dissolution testing. Most dissolution labs have many sets of baskets, often more than one set per dissolution apparatus, so it is not uncommon for even a medium-sized dissolution laboratory to have over 100 baskets. Each basket should be checked for damage and USP compliance before each dissolution test. If the basket shape or dimensions are out of compliance with USP requirements, the observed dissolution rate could be altered and could cause an erroneous dissolution result. These checks for dimension compliance and damage need to be conducted before each dissolution, since damage to the basket could occur at any time. Routine mechanical calibration (wobble) and chemical calibrator testing may resolve a noncompliant basket, but since most labs infrequently conduct these more extensive tests (typically every 6 months), a great many dissolution tests will have been conducted with the damaged basket by the time the mechanical or chemical calibration is executed.

In addition, basket dimension requirements are even more critical for robotic dissolution testing. Most robotic dissolution apparatus utilize an o-ring to hold the basket onto the shaft. If the top opening of the basket is not a true circle or is the wrong size, then the basket may not fit onto the o-ring, it may slip off, or it may damage the o-ring itself. This will lead to basket drops, where the basket falls off the shaft and into the dissolution bath before the start of the test, resulting in an aborted dissolution run. Additionally the robotic system that places the baskets on the shafts can, over time, damage the baskets, resulting in bent or warped baskets that could lead to erroneous dissolution results. Additionally, bent or distorted baskets can jam the feed mechanism of a robotic dissolution apparatus, again leading to aborted dissolution runs.

Clearly; control of the dimensions of the dissolution basket is critical to ensure USP compliance, accurate and consistent dissolution measurements, and efficient and robust robotic dissolution operation. 
Our laboratory has implemented the use of this Go-NoGo gauge to ensure that we control the dimensions of all dissolution baskets. The Go-NoGo gauge is used on all baskets before each dissolution test to ensure that there are no dimensional issues with our baskets. We combine this gauge check with a visual examination for rust or other obvious damage. This process, combined with our biannual dissolution calibration routine (mechanical and chemical calibrator) and other pre-run checks, ensures that our dissolution apparatus are in compliance with the requirements set forth in the USP.

The gauge itself checks many of the dimensions that are required by USP.The gauge controls basket height as well as the i.d. and o.d. of the top opening and bottom portion. In addition, it controls the cylindrical symmetry of the basket and ensures that there are no dents in the basket. This check also eliminates baskets that could cause a significant amount of wobble, which is specified in the USP.Wobble could come from the basket or the shaft. Our six-month mechanical calibration ensures that the shaft has an acceptable wobble, and the Go-NoGo gauge ensures that no damage to the basket has occurred between calibrations, which would result in an increase of this wobble level. Our empirical observations indicate that most wobble is caused by bent and asymmetric baskets, so the use of the gauge, combined with a periodic wobble check to ensure shaft symmetry, will eliminate most wobble issues.

The gauge itself is checked periodically against a calibrated caliper to ensure that the dimensions of each gauge are within a set specification. This process is specified in our general dissolution SOP.

The use of the gauge combined with visual observations and other routine pre-test checks (vessel centering, basket height) significantly increase the level of USP compliance and, therefore, the quality of the data generated in our dissolution laboratory.

Also, we have observed an increase in the robustness of our robotic dissolution systems since the gauge was implemented. The pre-run check of each basket with the gauge screens out baskets that would otherwise jam the basket feed mechanism or slip off the shaft.

The end result is that our dissolution apparatus operate more reliably, consistently, and with less day-to-day variability. This improves our ability to trend stability, enhances our overall productivity, reduces laboratory investigation reports, and improves our ability to transfer methods when the other lab utilizes the basket as well.

The gauge itself is extremely easy to utilize, and it takes only about one minute to check six baskets for a single dissolution. It takes less than 10 min to check a full complement of 48 baskets for a robotic dissolution experiment.

One consequence of this new gauge is that many of the baskets found in a dissolution lab will be identified as "non-optimal" and will be discarded. Thus, baskets will be replaced more frequently, which will result in an additional cost for the lab. This additional cost is minimal and justified given the additional quality, more accurate data, and more efficient robotic operation.

\section{CONCLUSION}

A simple, cost-effective, time-saving device has been invented to control the variability of a basket. This device not only controls the parameters set in USP $<711>$ (height, cylinder i.d., and o.d.) but also controls the cylinder symmetry, which is not defined in USP $<711>$.

\section{ACKNOWLEDGMENTS}

The authors would like to thank John Barlow, Paul Ameye, and Gregory Hywel for constructing the Go-NoGo gauge.

\section{REFERENCES}

1. Dissolution $<711>$. In United States Pharmacopeia and National Formulary USP 31-NF 26; The United States Pharmacopeial Convention, Inc.: Rockville, MD, 2007.

2. Dissolution test for solid dosage forms. In European Pharmacopoeia, 5th ed.; European Directorate for the Quality of Medicines, Council of Europe: Strasbourg, France, 2005.

3. Dissolution Test. In Japanese Pharmacopoeia, 15th ed.; Ministry of Health, Labour, and Welfare:Tokyo, Japan, 2007.

4. Kukura, J.; Baxter, J. L.; Muzzio, F. J. Shear distribution and variability in the USP apparatus 2 under turbulent conditions. Int. J. Pharm. 2004, 279, 9-17.

5. Healy, A. M..; McCarty, L. G.; Gallagher, K. M.; Corrigan, G. I. Sensitivity of dissolution rate to location in the paddle dissolution apparatus. J. Pharm. Pharmacol. 2002, 54, 441-444.

6. Mirza, T.; Joshi, Y.; Liu, G.; Vivilecchia, R. Evaluation of Dissolution Hydrodynamics in the USP, Peak ${ }^{\mathrm{TM}}$ and Flat-Bottom Vessels Using Different Solubility Drugs. Dissolution Technol. 2005, 12 (1), 11-16.

7. Gray, V.Identifying sources of error in calibration and sample testing. Am. Pharm. Rev. 2002, 5 (2), 8-13.

8. USP Informational General Chapter <1092> The Dissolution Procedure: Development and Validation. Pharm. Forum 2005, 31, 1463-1475.

9. Hanson, R; Gray V. Handbook of Dissolution Testing, 3rd ed.; Dissolution Technologies, Inc.: Hockessin, DE, 2004.

10. Dressman, J.; Kramer, J. Pharmaceutical Dissolution Testing; Taylor and Francis: Boca Raton, FL, 2005.

11. PhRMA Dissolution Calibration Subcommittee. Dissolution Calibration: Recommendations for Reduced Chemical Testing and Enhanced Mechanical Calibration. Pharm. Forum 2000, 26, 1149-1166.

12. Beyer, W.; Smith, D. Unexpected variable in the USP/NF rotating basket dissolution rate test.J.Pharm. Sci. 1971, 60, 2350-2351. 
13. Hanson, W. Solving the puzzle of random variables in dissolution testing. Pharm. Tech. 1977, 1, 30-41.

14. Collins, C. C. Vibration: What Is It and How Does It Affect Dissolution Testing? Dissolution Technol. 1998, 5 (4), 16-18.

15. Thakker, K.; Naik, N.; Gray, V.; Sun, S. Fine tuning of the dissolution apparatus. Pharm. Forum 1980, 6, 177-185.

16. Crist, B.; Spisak, D. Evaluation of Induced Variance of Physical Parameters on the Calibrated USP Dissolution Apparatus 1 and 2.Dissolution Technol. 2005, 12 (1), 28-34.

17. Scott, P. Geometric Irregularities Common to the Dissolution Vessel. Dissolution Technol. 2005, 12 (1), 18-21.

18. Cox, D. C.; Wells, C. E.; Furman, W. B.; Savage, T. S.; King, A. C. Systematic error associated with apparatus 2 of the USP dissolution test II: effect of deviations in vessel curvature from that of a sphere.J.Pharm. Sci. 1982, 71, 395-399.
19. Tanaka, M.; Fujiwara, H.; Fujiwara, M. Effect of the Irregular Inner Shape of a Glass Vessel on Prednisone Dissolution Results. Dissolution Technol. 2005, 12 (4), 15-19.

20. Baxter, J. L.; Kukura. J.; Muzzio, F. J. Hydrodynamicsinduced variability in the USP apparatus II dissolution test. Int. J. Pharm. 2005, 292, 17-28.

21. Vangani, S.; Flick, T.; Tamayo, G.; Chiu, R.; Cauchon, N. Vibration Measurements on Dissolution Systems and Effects on Dissolution of Prednisone Tablets RS. Dissolution Technol. 2007, 14 (1), 6-14.

22. Eaton, J.; Deng, G.; Hauck, W.; Brown, W. E.W.; Manning, R. G.; Wahab, S.Z. Perturbation Study of Dissolution Apparatus Variables-A Design of Experiment Approach. Dissolution Technol. 2007, 14 (1), 20-27.

23. Liddell, M.; Deng, G.; Hauck, W.W.; Brown, W.E.;Wahab, S. Z.; Manning, R. G. Evaluation of Glass Dissolution Vessel Dimensions and Irregularities. Dissolution Technol. 2007, 14 (1), 28-34. 